\title{
Esclerite: características clínicas, associação sistêmica, tratamento e evolução de 100 pacientes
}

\author{
Scleritis: clinical characteristics, systemic associations, treatment \\ and outcome in 100 patients
}

\author{
Danuza de Oliveira Machado ${ }^{1}$ \\ André Luis Land Curi² \\ Raphael Stehling' Fernandes ${ }^{3}$ \\ Thais Fontes Bessa ${ }^{4}$ \\ Wesley Ribeiro Campos ${ }^{5}$ \\ Fernando Oréfice $^{6}$
}

Trabalho realizado no serviço de Uveítes do Hospital São Geraldo do Hospital das Clínicas da Universidade Federal de Minas Gerais - UFMG - Belo Horizonte (MG) - Brasil.

${ }^{1}$ Pós-graduanda (doutorado) pela Universidade Federal de Minas Gerais - UFMG - Belo Horizonte (MG), Brasil.

${ }^{2}$ Doutor em Oftalmologia pela UFMG - Belo Horizonte (MG) - Brasil.

${ }^{3}$ Assistente do Setor de Uveítes do Hospital São Geraldo do Hospital das Clínicas da UFMG - Belo Horizonte (MG) - Brasil.

${ }^{4}$ Assistente do Setor de Uveítes do Hospital São Geraldo do Hospital das Clínicas da UFMG - Belo Horizonte (MG) - Brasil

${ }^{5}$ Chefe do Setor de Uveítes do Hospital São Geraldo do Hospital das Clínicas da UFMG - Belo Horizonte (MG) - Brasil.

${ }^{6}$ Livre-docente, Professor Titular de Oftalmologia da UFMG - Belo Horizonte (MG) - Brasil.

Endereço para correspondência: Danuza de Oliveira Machado. Rua Professor Raimundo Cândido, 130/602 - Belo Horizonte (MG) CEP 30320-650

E-mail: danuzadeoliveira@ig.com.br

Recebido para publicação em 28.11.2006 Última versão recebida em 06.12 .2008 Aprovação em 16.02.2009

Nota Editorial: Depois de concluída a análise do artigo sob sigilo editorial e com a anuência da Dra. Áisa Haidar Lani sobre a divulgação de seu nome como revisora, agradecemos sua participação neste processo.

\begin{tabular}{|l|}
\hline RESUMO \\
\hline Objetivos: Documentar características clínicas, associações sistêmicas, \\
tratamento e evolução de 100 pacientes com esclerite, examinados no \\
serviço de uveítes da Universidade Federal de Minas Gerais. Pacientes \\
e Métodos: Identificados 100 pacientes com esclerite, registrados e \\
analisados dados com relação às queixas dos mesmos, sinais oculares, \\
visão, alterações ecográficas, manifestações sistêmicas, tratamento e \\
evolução. Resultados: Sessenta e nove pacientes eram mulheres e 31 \\
homens. Esclerite anterior difusa e nodular ocorreu em 71 pacientes, \\
esclerite anterior necrosante em 3, esclerite posterior em 24 e escle- \\
romalácia perfurans em 2 pacientes. Envolvimento unilateral em 79 e \\
bilateral em 21 pacientes. A principal queixa foi dor ocular e o sinal \\
fundoscópico predominante na esclerite posterior foi o descolamento \\
seroso de retina. Em 13 pacientes a esclerite determinou o encontro de \\
doença sistêmica e a principal forma de tratamento foi com droga anti- \\
inflamatória não-esteróide oral. Dezoito pacientes precisaram de trata- \\
mento imunossupressor para o controle do quadro ocular e a incidência \\
de complicação ocular foi de 35\%. Discussão: Esclerite é doença rara, às \\
vezes de difícil diagnóstico e potencialmente devastadora, todos os \\
esforços devem ser necessários para um diagnóstico rápido e correto \\
dessa doença. O conhecimento sobre a esclerite, suas formas de apresen- \\
tação, associações sistêmicas, tratamento e evolução são fundamentais \\
para que possamos fazer este diagnóstico correto e conduzir o quadro \\
ocular da maneira mais adequada possível tendo sempre como objetivo \\
final o controle do quadro escleral e preservação da visão do paciente. \\
\hline
\end{tabular}

Descritores: Esclerite/quimioterapia; Infecções oculares; Ciclosporinas/uso terapêutico

\section{INTRODUÇÃO}

A esclerite é uma doença inflamatória ocular que ainda hoje, apresenta diagnóstico difícil. A grande maioria dos casos é leve e idiopático, respondendo de maneira satisfatória ao uso de drogas anti-inflamatórias orais. Por outro lado, podem ocorrer apresentações mais agressivas e não responsivas a terapêutica convencional aumentando o risco de complicações oculares graves, perda visual e associação com doença sistêmica.

A esclerite geralmente compromete indivíduos de meia idade, sua principal forma de apresentação é a esclerite anterior e a dor ocular é seu sintoma predominante ${ }^{(1)}$. O quadro ocular pode ser idiopático ou estar associado a doenças sistêmicas ${ }^{(2)}$. Dentre as doenças mais frequentemente associadas à inflamação escleral podemos citar doenças infecciosas: herpes zoster 
oftálmico, herpes simples, sífilis, tuberculose (TBC); doenças reumáticas como artrite reumatóide e lúpus eritematoso sistêmico (LES); vasculites sistêmicas como granulomatose de Wegener, poliarterite nodosa, arterite de células gigantes e doença inflamatória intestinal como reto-colite-ulcerativa e doença de $\mathrm{Crohn}^{(2-3)}$. Infecções e malignidades devem ser excluídas. O risco de associação sistêmica é maior em pacientes idosos e na presença de esclerite anterior necrosante com inflamação ${ }^{(4)}$.

A avaliação diagnóstica é importante em todos os casos de esclerite e inclui avaliação clínica geral, com interrogatório dos sistemas além de investigação laboratorial. O encaminhamento para o reumatologista é aconselhável para cooperação no diagnóstico e tratamento.

O tratamento depende do diagnóstico correto e, em casos mais graves e não responsivos ao tratamento convencional com drogas anti-inflamatórias orais, pode ser necessário o uso de drogas imunossupressoras para o controle do quadro ocular.

A cada ano, novos relatos sobre associações e apresentações atípicas da esclerite são divulgados tornando esse diagnóstico cada vez mais difícil e exigindo elevado índice de suspeita para detectarmos essa doença que pode ser potencialmente destrutiva.

O objetivo deste estudo é documentar as características clínicas, associações sistêmicas, tratamento e evolução de 100 pacientes com esclerite, atendidos no serviço de uveítes do Hospital São Geraldo-HC-UFMG no período de janeiro de 2001 até agosto de 2006.

\section{MÉTODOS}

Uma revisão retrospectiva dos prontuários médicos de todos os pacientes com esclerite, atendidos nos serviço de uveítes da Universidade Federal de Minas Gerais entre os anos de 2001 e 2006, foi realizada. Foram identificados 100 pacientes e todos, foram incluídos no trabalho. O diagnóstico de esclerite foi utilizado como critério de inclusão dos pacientes neste trabalho. Não houve critérios de exclusão.

Detalhes com relação ao início da doença, classificação da esclerite (de acordo com a classificação proposta por Watson e Hayreh em 1976) ${ }^{(5)}$, queixas dos pacientes, sinais e sintomas clínicos e oculares, alterações na ecografia $B$, associação com doença sistêmica, tratamento e evolução do quadro foram registrados e analisados pelo programa estatístico SPSS (análise descritiva - frequência). Os dados dos pacientes foram coletados em uma única ocasião (corte transversal) e analisados ao longo de três meses (de junho de 2006 a setembro de 2006). A data final para a inclusão de novos pacientes foi $30 \mathrm{de}$ setembro de 2006.

O trabalho foi aprovado pelo Departamento de Oftalmologia-Otorrinolaringologia e Fonoaudiologia e também foi aprovado pelo Comitê de Ética em Pesquisa da UFMG (COEP) sob o parecer número ETIC 244/07.

\section{RESULTADOS}

Dentre os 100 pacientes que participaram desse estudo, $69 \%$ eram do sexo feminino e $31 \%$ do sexo masculino sendo que, $43 \%$ desses pacientes tinham entre 41 e 59 anos de idade; $32 \%$ entre 21 e 40 anos; $22 \%$ eram maiores de 60 anos e apenas $3 \%$ dos pacientes tinham menos de 20 anos. A média de idade dos pacientes com esclerite foi de 48 anos.

O quadro ocular comprometeu ambos os olhos, de maneira simultânea ou não, em $21 \%$ dos casos. Em $79 \%$ dos casos, a doença escleral ocorreu de maneira unilateral comprometendo principalmente o olho direito ( $42 \%$ dos casos).

Com relação à classificação da esclerite (Tabela 1), 38\% dos 100 pacientes apresentaram um quadro de esclerite anterior nodular; $33 \%$ de esclerite anterior difusa; $1 \%$ de esclerite anterior necrosante após cirurgia ocular; $2 \%$ de esclerite anterior necrosante com inflamação; $2 \%$ de esclerite anterior necrosante sem inflamação ocular; $14 \%$ dos pacientes apresentaram esclerite posterior isolada e $10 \%$, esclerite posterior associada à esclerite anterior ( 7 com esclerite anterior difusa, 2 com esclerite anterior nodular e $1 \mathrm{com}$ esclerite anterior necrosante).

Houve mudança de apresentação do quadro escleral em 3 pacientes: 1 paciente evoluiu de esclerite anterior difusa para esclerite anterior nodular e 2 pacientes evoluíram para esclerite anterior necrosante com inflamação.

O quadro ocular foi recorrente em $41 \%$ dos casos e as duas queixas oculares mais frequentes foram dor ocular $(97 \%)$ e hiperemia (86\%). Dor a movimentação ocular foi citada por $13 \%$ dos pacientes e $26 \%$, notaram embaçamento visual.

Dentre os 24 pacientes com esclerite posterior o sinal clínico mais comum foi o descolamento seroso de retina em 17 pacientes seguido pelo edema de disco óptico em 6 pacientes e infiltrados sub-retinianos também em 6 pacientes. A principal alteração ecográfica na esclerite posterior foi o espessamento esclerocoroidiano em 19 pacientes. Em apenas 7 dos 24 pacientes com esclerite posterior foi constatado o clássico, mas não patognomônico, sinal do $\mathrm{T}$ (fluído no interior da cápsula de Tenon e na bainha do nervo óptico determinando uma letra $\mathrm{T}$ escura na ecografia. A barra horizontal do $\mathrm{T}$ é

\begin{tabular}{|lrc|}
\hline \multicolumn{3}{|c|}{ Tabela 1. Classificação das esclerites } \\
Classificação das esclerites & Frequência & $\begin{array}{c}\text { Porcentual } \\
\text { (\%) }\end{array}$ \\
Esclerite anterior difusa e posterior & 7 & $7,0 \%$ \\
Esclerite anterior necrosante e posterior & 1 & $1,0 \%$ \\
Esclerite anterior necrosante pós cirurgia & 1 & $1,0 \%$ \\
Esclerite anterior nodular e posterior & 2 & $2,0 \%$ \\
Esclerite anterior difusa & 33 & $33,0 \%$ \\
Esclerite anterior necrosante & 2 & $2,0 \%$ \\
Esclerite anterior nodular & 38 & $38,0 \%$ \\
Esclerite posterior & 14 & $14,0 \%$ \\
Escleromalácia perfurans & 2 & $2,0 \%$ \\
Total & 100 & $100,0 \%$ \\
\hline
\end{tabular}


formada pela ecoluscência do fluído na cápsula de Tenon e a barra vertical pela ecoluscência do disco óptico de aspecto dilatado).

Setenta e oito dos 100 pacientes com esclerite (78\% dos casos) não apresentavam doença sistêmica previamente diagnosticada. Sete pacientes apresentavam artrite reumatóide (7\%); havia 5 pacientes com sífilis (5\%); 2 com tuberculose (2\%); 2 com gota (2\%); 1 com febre reumática (1\%); 1 com doença de Crohn (1\%); 1 com retocolite ulcerativa (1\%); 1 com LES (1\%); 1 com hanseníase (1\%) e 1 paciente com síndrome de Sjögren (1\%) (Gráfico 1).

A esclerite determinou o encontro de doença sistêmica em 13 dos 100 pacientes (13\% dos casos): síndrome de Cogan em 1 paciente, espondilite anquilosante em 1 , herpes em 2 , granulomatose de Wegener em 3, sarcoidose em 1, policondrite recorrente em 1 e tuberculose em 4 pacientes. Em uma paciente, a esclerite foi secundária a uma infecção por Aspergillus sp (confirmada por biópsia escleral) (Gráfico 2).

O tratamento inicial foi com droga anti-inflamatória não esteróide oral (DAINE) em 53\% dos pacientes e em 38\%, foi utilizado o corticóide oral. Três pacientes, 1 com esclerite anterior necrosante pós-cirurgia de pterígio e 2 com esclerite associada à proptose grave (pseudotumor inflamatório) iniciaram a terapêutica com pulsoterapia de metilprednisolona. Um paciente com esclerite anterior nodular iniciou o tratamento com pulsoterapia de ciclofosfamida já que o mesmo era diabético e nos primeiros dias do uso de corticóide oral houve uma descompensação do diabetes. Duas pacientes com esclerite anterior necrosante iniciaram o tratamento com corticóide oral e poucos dias depois foi introduzida a azatioprina com excelente resposta terapêutica. Em uma paciente com retocolite ulcerativa e esclerite posterior que já estava em uso de azatioprina, foi necessária a introdução do corticóide oral para o

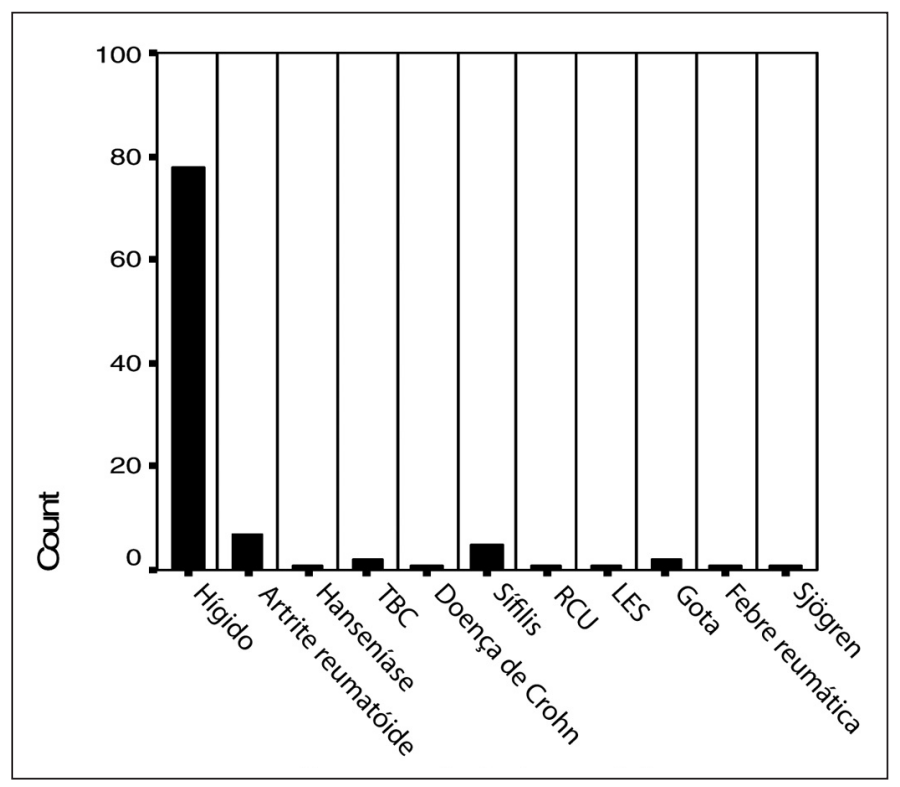

Gráfico 1 - Doenças sistêmicas prévias controle do quadro ocular. Um dos pacientes com granulomatose de Wegener, antes de receber o seu diagnóstico, iniciou o tratamento com corticóide oral, seguido de pulsoterapia venosa com metilprednisolona e ciclosporina oral (apresentava quadro bilateral e grave de esclerite anterior e posterior). Houve boa resposta com melhora do quadro ocular, mas após o diagnóstico, o seu tratamento foi modificado para pulsoterapia venosa com ciclofosfamida devido ao aparecimento de quadro pulmonar. Em um paciente com diagnóstico de herpes, o corticóide oral foi associado ao aciclovir oral e em outro paciente o corticóide oral foi associado a um antifúngico devido ao diagnóstico por biópsia de uma esclerite por Aspergillus.

A resposta à terapêutica inicial foi satisfatória em $45 \%$ dos casos com resolução rápida e total do quadro ocular. Em 68\% dos casos, não foi necessária a modificação da terapêutica inicial. Abandono do tratamento foi constatado em três pacientes. Em 29\% dos casos foi necessária a modificação da terapêutica inicial: 17\% de DAINE oral para corticóide oral; $3 \%$ para pulsoterapia de ciclofosfamida após diagnóstico de granulomatose de Wegener; $1 \%$ para pulsoterapia de ciclofosfamida seguida do uso de ciclosporina oral (a paciente apresentava esclerite anterior necrosante e não respondeu ao uso do corticóide oral); $2 \%$ para ciclosporina oral e 5\% para a azatioprina. A paciente que apresentou esclerite associada ao Aspergillus sp evoluiu para enucleação do olho comprometido. Após a modificação do tratamento houve controle do quadro ocular em todos os pacientes.

Em 92 pacientes não houve complicação do tratamento sistêmico. Hipertensão arterial foi a principal complicação sistêmica exigindo a suspensão do tratamento em dois pacientes.

Com relação às complicações da esclerite, $65 \%$ dos pacientes evoluíram sem complicações; 9 pacientes apresentaram

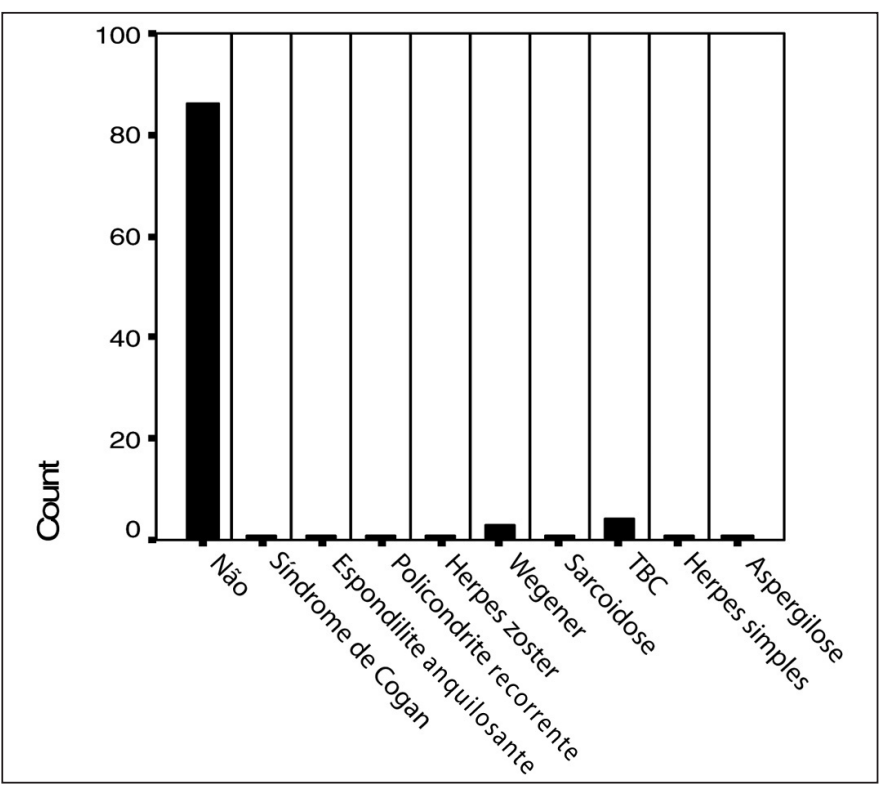

Gráfico 2 - Diagnóstico de doença sistêmica 
afilamento escleral; catarata foi constatada em 6 pacientes; afilamento corneano em 2; aumento da transparência escleral em 5; glaucoma em 8; baixa visual permanente em 3; uveíte em 2 e perfuração escleral em 1 paciente. Quarenta e sete pacientes ainda recebiam tratamento até o fechamento deste estudo.

\section{DISCUSSÃO}

No estudo apresentado, 100 pacientes foram incluídos com o diagnóstico de esclerite. A grande maioria dos pacientes (43\%) tinha entre 41 a 59 anos de idade, quando apresentou o primeiro episódio da doença o que é compatível com vários outros estudos que mostram uma idade média de 48 anos para o início das esclerites ${ }^{(6-8)}$. Alguns estudos mostraram que aqueles que desenvolvem a doença escleral com mais de 60 anos apresentam um risco significativamente maior para doença mais agressiva, complicações e perda visual ${ }^{(6,9-11)}$.

A esclerite é duas vezes mais comum em mulheres do que em homens ${ }^{(12)}$, no nosso estudo, $69 \%$ eram mulheres o que está de acordo com a literatura. O quadro ocular pode se tornar bilateral em $1 / 3$ dos pacientes sendo o segundo olho envolvido de três meses a seis anos após o envolvimento do primeiro olho $^{(13)}$. Vinte e um pacientes $(21 \%)$ apresentavam quadro ocular bilateral.

Apesar da primeira descrição de uma esclerite grave ter sido realizada em 1717 , foi só depois de $1963^{(5)}$, quando se instalou um setor específico para o estudo dessa doença no Moorfields Eye Hospital em Londres, que as divisões e subdivisões da doença escleral e episcleral foram identificadas. Atualmente a classificação mais completa divide a esclerite em esclerite anterior difusa; nodular; necrosante com inflamação (granulomatosa, vaso-oclusiva e cirurgicamente induzida); necrosante sem inflamação (escleromalácia perfurans) e esclerite posterior (difusa, nodular e necrosante) ${ }^{(1)}$. Cerca de $70 \%$ dos pacientes do presente estudo apresentaram um quadro de esclerite anterior difusa e nodular, que são as duas principais formas de apresentação da doença, sendo a primeira mais benigna que a segunda. Esclerite anterior necrosante foi constatada em quatro pacientes sendo que em um caso, havia relato de cirurgia ocular prévia (cirurgia de pterígio). Dois pacientes apresentaram escleromalácia perfurans associada à artrite reumatóide (sua mais comum associação) ${ }^{(14)}$ e 24 pacientes evoluíram com esclerite posterior doença que até recentemente era considerada rara, mas hoje se sabe que ela pode estar sendo pouco diagnosticada. Estudos de ultrassonografia B mostram que a esclerite posterior é quase tão comum quanto à esclerite anterior ${ }^{(15)}$.

Mudança de apresentação do quadro escleral ocorreu em três pacientes, entretanto vários estudos têm revelado que quando a esclerite recorre, ela quase sempre o faz na mesma forma da sua apresentação original ${ }^{(2)}$.

Com relação à sintomatologia, a esclerite é considerada uma doença ocular extremamente dolorosa. Dor ocular é a sua manifestação predominante embora possa estar ausente em casos de esclerite posterior e escleromalácia perfurans ${ }^{(2)}$. A dor pode ser tão intensa a ponto de acordar o paciente durante o sono e a esclerite é uma das poucas doenças oculares que provoca esse tipo de sintomatologia. Dor ocular intensa foi relatada por $97 \%$ dos pacientes deste estudo.

A esclerite sendo uma doença inflamatória ocular, frequentemente está associada a complicações oculares e doenças sistêmicas $^{(2,4,14,16)}$. Vários relatos de séries de casos têm mostrado que 40 a $50 \%$ dos pacientes com esclerite apresentam uma doença infecciosa ou reumatológica associada, sendo esta, a principal associação ${ }^{(17-18)}$. Algumas doenças reumatológicas descritas em associação com a esclerite incluem a artrite reumatóide, LES, vasculites sistêmicas tais como a granulomatose de Wegener, policondrite recorrente e doença inflamatória intestinal. Na esclerite anterior necrosante o risco de associação com doença sistêmica é cerca de $95 \%{ }^{(7)}$, dessa maneira, todo paciente que apresentar um quadro de grave de esclerite, refratário ao tratamento ou recorrente deverá ser investigado para afastar a presença de doença sistêmica. Na nossa série de casos a esclerite determinou o encontro de doença sistêmica em 13 dos 100 pacientes. Sete pacientes já apresentavam diagnóstico prévio de artrite reumatóide. A porcentagem total de pacientes com esclerite e associação sistêmica foi de $36 \%$.

A proposta do tratamento da esclerite é de suprimir ou eliminar a resposta inflamatória com o mínimo de medicação possível. A maioria dos pacientes com esclerite anterior difusa ou nodular podem ser tratados com drogas anti-inflamatórias não-esteróides administradas por via oral ${ }^{(1,6,9)}$. Se o tratamento não for efetivo em 10 dias ou se o paciente apresentar esclerite posterior, prednisona oral deverá ser iniciada com uma dosagem de 60 - $80 \mathrm{mg} /$ dia seguida de redução progressiva até $20 \mathrm{mg} /$ dia tão logo haja o controle do quadro ocular. Um total de 53 pacientes recebeu como terapêutica inicial o naproxeno oral evoluindo com melhora do quadro ocular na grande maioria dos casos (não foi necessária a mudança desse tratamento inicial em $68 \%$ dos pacientes). Em apenas $32 \%$ foi necessária a substituição do naproxeno oral pelo corticóide oral.

Pacientes com esclerite anterior necrosante e aqueles com outras formas de esclerite persistente e grave vão requerer tratamento com prednisona oral em associação com drogas imunossupressoras tais como o methotrexate, ciclofosfamida, ciclosporina, azatioprina e outras medicações ${ }^{(2)}$. Pulsoterapia de ciclofosfamida foi realizada em cinco dos nossos pacientes: três após o diagnóstico de granulomatose de Wegener, outro como terapêutica inicial para uma esclerite anterior nodular grave, diabético e com passado de descontrole da diabetes pelo uso de corticóide oral e o quinto paciente, com diagnóstico de esclerite anterior necrosante. Este último paciente controlou de maneira parcial o seu quadro ocular sendo necessária à introdução de ciclosporina oral que foi mantida por alguns anos após o total controle do quadro. A pulsoterapia de ciclofosfamida é preferida em relação à pulsoterapia de metilprednisolona se diagnóstico de granulomatose de We- 
gener ${ }^{(19-20)}$. A ciclosporina oral tem se mostrado bastante eficaz no tratamento das esclerites graves ou refratárias ao tratamento usual ${ }^{(21)}$. Mais dois pacientes necessitaram do uso de ciclosporina oral e sete pacientes mais idosos (mais de 50 anos) e com contraindicação para o uso da ciclosporina, conseguiram controlar o quadro escleral após a introdução da azatioprina. Duas delas apresentavam esclerite anterior necrosante grave sendo uma associada à sarcoidose.

As principais complicações da esclerite são: envolvimento corneano, catarata, glaucoma, uveítes e aqueles com esclerite posterior estão em maior risco de perda visual por envolvimento direto da retina e disco óptico ${ }^{(11)}$. Em um estudo realizado a incidência de complicações oculares associadas à esclerite chegou a $58,8 \%{ }^{(6)}$, no presente trabalho essa incidência foi de $36 \%$. Dentre as complicações encontradas na nossa série podemos citar o afilamento da esclera e córnea, catarata e glaucoma.

Embora a doença escleral seja uma doença rara, a esclerite anterior necrosante com inflamação pode ser uma doença extremamente grave e destrutiva levando à perda visual definitiva; então, todos os esforços devem ser necessários para um diagnóstico rápido e correto dessa doença. $\mathrm{O}$ conhecimento sobre a esclerite, suas formas de apresentação, associações sistêmicas, tratamento e evolução são fundamentais para que possamos fazer este diagnóstico correto e conduzir o quadro ocular da maneira mais adequada possível tendo sempre como objetivo final o controle do quadro escleral e preservação da visão do paciente.

\section{ABSTRACT}

Purpose: To document the clinical features, systemic association, treatment and evolution of 100 patients with scleritis evaluated at the Uveitis Service of the Federal University of Minas Gerais. Patients and Methods: 100 patients were identified with the diagnosis of scleritis. Signals and symptoms, visual acuity, B-mode ultrasonography signals, systemic associations, treatment and evolution were described and analyzed. Results: 69 patients were female and 31 were male. Diffuse and nodular anterior scleritis occurred in 71 patients, necrotizing anterior scleritis in 3, posterior scleritis in 24 and escleromalacia perforans in 2 patients. Unilateral involvement occurred in 79 patients and bilateral involvement in 21 patients. The main symptoms were ocular pain and redness and the main signal in posterior scleritis was the serous detachment of the retina. Scleritis in association with systemic disease occurred in 35 patients and the principal kind of treatment was the use of oral NSAIDs. Only 18 patients required systemic immunosuppressive drugs. Ocular complications were detected in 35 patients. Conclusions: Scleritis may represent a diagnostic challenge and is often associated with life threatening systemic disease and vision threatening ocular complications. Knowledge of scleritis may aid in determining timely and accurate diagnosis and treatment of both the ocular and any associated systemic conditions, thus decreasing morbidity and mortality.

Keywords: Scleritis/drug therapy; Eye infections; Cyclosoporins/therapeutic use

\section{REFERÊNCIAS}

1. Watson PG. The management of inflammatory scleral disease. Contemp Ophthalmol. 2003;2:1-7.

2. Watson PG, Hazleman BL, Pavesio CE, Green WR. The sclera and systemic disorders. $2^{\text {nd }}$ ed. Philadelphia:WB Saunders; 2004.

3. Oréfice F, Pavésio CE. Esclerites. In: Oréfice F. Uveíte: clínica e cirúrgica: texto e atlas. $2^{\underline{a}}$ ed. Rio de Janeiro: Cultura Médica; 2005. Vol.2. p.977-86.

4. Pavesio CE, Meier FM. Systemic disorders associated with episcleritis and scleritis. Curr Opin Ophthalmol. 2001;12(6):471-8.

5. Watson PG, Hayreh SS. Scleritis and episcleritis. Br J Ophthalmol. 1976;60(3): 163-91.

6. Jabs DA, Mudun A, Dunn JP, Marsh MJ. Episcleritis and scleritis: clinical features and treatment results. Am J Ophthalmol. 2000;130(4):469-76.

7. Sainz de la Maza M. Episcleritis and scleritis. Contemp Ophthalmol. 2006; 5(9):1-8.

8. Tuft SJ, Watson PG. Progression of scleral disease. Ophthalmology. 1991;98(4): 467-71.

9. Okhravi N, Odufuwa B, McCluskey P, Lightman S. Scleritis. Surv Ophthalmol. 2005;50(4):351-63. Review.

10. Sainz de la Maza M, Jabbur NS, Foster CS. Severity of scleritis and episcleritis. Ophthalmology. 1994;101(2):389-96.

11. Watson PG, Young RD. Scleral structure, organisation and disease. A review. Exp Eye Res. 2004;78(3):609-23.

12. Watson PG. Disease of sclera and episclera. In: Tasman W, Jaeger EA, editors. Duane's clinical ophthalmology. Philadelphia: Lippincott-Raven; 1992.

13. Watson PG. Clinical presentations of scleritis and episcleritis. In: Watson PG, Hazleman BL, Pavesio CE, Green WR. The sclera and systemic disorders. London: Butterworth-heinemann; 2004. p.60-88.

14. Sainz de la Maza M, Foster CS, Jabbur NS. Scleritis associated with rheumatoid arthritis and with other systemic immune-mediated diseases. Ophthalmology. 1994;101(7):1281-6; discussion 1287-8

15. McCluskey PJ, Watson PG, Lightman S, Haybittle J, Restori M, Branley M. Posterior scleritis: clinical features, systemic associations, and outcome in a large series of patients. Ophthalmology. 1999;106(12):2380-6.

16. Sainz de la Maza M, Foster CS, Jabbur NS. Scleritis associated with systemic vasculitic diseases. Ophthalmology. 1995;102(4):687-92.

17. Akpek EK, Thorne JE, Qazi FA, Do DV, Jabs DA. Evaluation of patients with scleritis for systemic disease. Ophthalmology. 2004;111(3):501-6. Comment in: Ophthalmology. 2007;114(6):1232.

18. Akpek EK, Uy HS, Christen W, Gurdal C, Foster CS. Severity of episcleritis and systemic disease association. Ophthalmology. 1999;106(4):729-31.

19. Arnavaz AB, Freissler K, Lang GE. [Posterior scleritis in Wegener disease]. Ophthalmologe. 1997;94(8):595-9. German

20. McCluskey P, Wakefield D. Intravenous pulse methylprednisolone in scleritis. Arch Ophthalmol. 1987;105(6):793-7.

21. Wakefield D, McCluskey P. Cyclosporin therapy for severe scleritis. Br J Ophthalmol. 1989;73(9):743-6. 